\title{
Analog Network Coding for Multi-User Multi-Carrier Differential Chaos Shift Keying Communication System
}

\author{
Georges Kaddoum* and Farhad Shokraneh
}

\begin{abstract}
This paper presents the design and performance analysis of an Analog Network Coding (ANC) scheme for multiuser Multi-Carrier Differential Chaos Shift Keying (MC-DCSK) modulation. The incentives to employ MC-DCSK system are to achieve a better spectral efficiency and more efficient energy consumption compared to that of a conventional DCSK system. The proposed scheme considers a network comprising $L$ user nodes $(L \geq 2)$, and a single relay node $R$. In this scheme, called ANC-based two-way relay system, all users transmit their signals to the relay in the first time slot, and the relay forwards the superposition of the received signals each of the user nodes in the second time slot. At the receiver end, each user mitigates the overall interference by subtracting its own data signal from the received combined signal, and then starts the decoding process. The system design is analytically studied and the corresponding theoretical bit error rate expression for the multipath fading channel is derived. Additionally, the conventional ANC-DCSK scheme is analyzed and compared to the proposed ANC-based MC-DCSK scheme to show the improvement in the performance of our approach. Finally, to validate the accuracy of the methodology, the simulation results are compared to the related theoretical expressions.
\end{abstract}

Index Terms-Non-coherent chaos based communication system, Multi-carrier DCSK, Analog network coding, Performance analysis.

\section{INTRODUCTION}

$\mathbf{I}$ $\mathrm{T}$ has been envisioned by some studies that there will be over seven trillion wireless devices serving seven billion people by 2020 [1]. In this sense, the development of advanced methods to mitigate the interference imposed by different user signals is indispensable. This interference are often treated as a destructive phenomenon in many networks. However, the analog network coding (ANC) proposed in [2] is an example that exploits the interference to increase the network capacity [3].

Analog network coding (ANC) is a new spectrally efficient technique for multi-way or two-way relay channels [2], [4]. In this network scheme, two user nodes simultaneously transmit their data signals to the relay in the first time slot. In the second time slot, the relay node forwards the superimposed version of these signals to the user nodes. The ANC scheme harnesses the interference originating from the linear combination of the transmitted user signals which naturally add in the physical

G. Kaddoum and F. Shokraneh are with University of Québec, ETS, LaCIME Laboratory, 1100 Notre-Dame west, H3C 1K3, Montreal, Canada (e-mail: georges.kaddoum@etsmtl.ca; farhad.shokraneh@lacime.etsmtl.ca)

* This work has been supported by the NSERC discovery grant $435243-$ 2013.

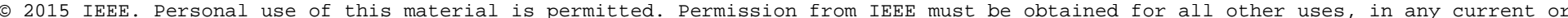

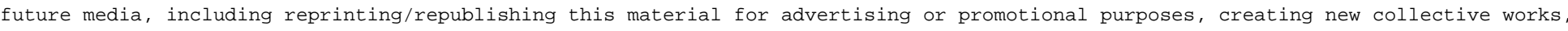
for resale or redistribution to servers or lists, or reuse of any copyrighted component of this work in other works.

DOI : $10.1109 /$ TWC. 2014.2367508 channel. Therefore, not only the destructive effect of the interference is reduced or eliminated, but also the capacity of the networks is boosted. It has also been shown that this method is an efficient technique to reduce the required number of forwarding operations and also to increase the throughput of the system [5]. Recently, a growing number of research have been conducted on ANC-based coherent spread spectrum systems (CDMA) [6]-[8]. Although simple, the ANC scheme has proved to offer a considerably better performance and less sensitivity to synchronization compared to that of the physical network coding schemes [3].

The reason for applying the chaos-based modulations to the ANC technique lies in the significant advantages provided by this modulation schemes [9], [10]. Basically, chaotic systems due to their high sensitivity upon initial conditions have the potential to generate a theoretically infinite number of very low cross-correlated signals. Owing to their wideband characteristics, chaotic signals have proved to be one of the natural candidates for multi-user spread-spectrum modulation schemes [11]-[15]. Some of these chaos-based modulations provide the same advantages as conventional spread-spectrum modulations do, including mitigation of fading in varying channels [16], jamming resistance along with low probability of interception (LPI) [17], and secure communications [18]. Additionally, many studies have been done on reducing multiuser interference and the peak-to-average power ratio (PAPR), showing that chaos-based sequences outperform Gold and many other independent, identically distributed sequences in multi-user spread-spectrum communication systems [19], [20].

Among the digital chaos-based modulation schemes that have been proposed, chaos shift keying (CSK) and differential CSK (DCSK) have recently attracted a great deal of attention in the field of communication networks [21]-[23]. DCSK system has shown to be robust and easy to implement, although it requires a simple transceiver [24]. In this system due to the use of non-coherent receiver, the demodulation scheme is carried out without any need for generating the chaotic sequences. It has also been stated that this system performs well in multipath channels [16], [25] as well as in fast frequency and timevarying channels [26], [27]. In this respect, the performance of DCSK communication systems was studied in [28] for fading channels, and in [29]-[31] for cooperative schemes. Moreover, in [32], the transmission security of a DCSK system was improved. Given these advantages, some ultra-wideband (UWB) systems based on DCSK and FM-DCSK modulations have been proposed for Wireless Personal Area Networks 
(WPAN) [33]-[35].

However, DCSK scheme suffers from lower energy efficiency and data rate compared to that of conventional modulation schemes. This is mainly associated with the fact that half of the bit frame in this system is allocated to sending the non-information-bearing reference samples [11].

Many studies have been conducted to improve the spectral efficiency of DCSK schemes [36]-[38]. A new promising design of a multi-carrier DCSK system (MC-DCSK) for ultra-wideband systems is proposed in [39], and extended to a multi-user scenario in [40] which is a hybrid scheme with multi-carrier and DCSK modulations. This technique, in addition to the advantages of a DCSK system, benefits from a higher resistance to interference, higher data rate, and low transmission energy compared to that of the DCSK system.

Contributions: This paper presents a new bandwidth efficient ANC-based MC-DCSK system. In this new design, each user has an assigned private frequency for transmitting the reference signal, while the other available frequencies are shared among all user nodes for carrying the transmitted data symbols. The proposed scheme is a two-way relay system in which $L$ user nodes communicate with each other via an intermediate relay node. The communication process is carried out over two time slots. In the first time slot, or the multiple access phase, the user nodes transmit their signals to the relay synchronously or asynchronously. During the second time slot, or the broadcast phase, the relay forwards the superposition of the received signals to the users. Each user, after reducing the interference by subtracting its own data signal from the received signal, decodes the data of its interested user. It should be underlined that in a scenario of two user nodes $(L=2)$, the system performs without multi-user interference.

The novel contributions of this paper are summarized as follows:

1) The conventional ANC-based DCSK system is studied and its drawbacks and limitations are pointed out.

2) The proposed scheme is an extended version of the presented MC-DCSK system in [39] which employs multi-user multiple access technique with less bandwidth requirement than the system proposed in [40].

3) A new design of ANC scheme is applied to a noncoherent spread spectrum communication system based on chaotic modulation which can perform with more than two users.

4) In order to address the interference problem, an interference mitigating circuit is implemented at the receiver side which helps each user exclude its own data signal from the received signal before decoding its desired user data.

5) Finally, the corresponding interference level as well as the analytical end-to-end bit error ratio expressions over a multipath fading channel is derived and analyzed.

This paper is organized as the followings: In section II, the conventional DCSK system is explained and the architecture of the ANC-based DCSK and its major drawbacks and limitations are investigated. Section III presents the MC-DCSK model and proposes the application of the analog network coding to this system. Once the system is designed, the performance of the ANC-based MC-DCSK system is analyzed and the corresponding bit error rate expression under AWGN and multipath fading channels is derived in section IV. Section V is allocated to the simulation results that confirm the accuracy of the derived bit error rate expression. Finally, the related concluding remarks are discussed in section VI.

\section{ANC-BASED DCSK COMMUNICATION SYSTEMS}

This section starts with a brief description of DCSK system, and then it is shown how this scheme can be extended to an ANC-based DCSK scheme. Furthermore, the major drawbacks and limitations of this system are pointed out in order to highlight the advantages of the proposed MC-DCSK scheme as the potential candidate for the ANC protocol. To avoid confusion and for simplicity, the mathematical variables in $\mathrm{X}_{k, i, u, l, v}$ are chosen where $\mathrm{X}$ denotes the main variable, and the first lower index represents the sample index, whereas the second, third, fourth and the fifth one represent the bit, the data stream, the user, and the path indexes, respectively. Similarly, the same order is kept for the variables which have less than four lower indexes, e.g. $\mathrm{X}_{i, u, l}$ or $\mathrm{X}_{u, l}$, etc.

\section{A. DCSK Communication System}

As shown in Fig. 1, for user $l$ within the modulator each bit $s_{i, l}=\{-1,+1\}$ is represented by two sets of chaotic signal samples, where the first is allocated to the reference, and the second to the carrying data symbols. If +1 is transmitted, the data-bearing sequence is equal to the reference sequence, and if -1 is transmitted, an inverted version of the reference sequence is used as the data-bearing sequence. The spreading factor in the DCSK system is defined as the number of chaotic samples sent for each bit and presented by $2 \beta$ where $\beta$ is an integer. $T=2 T_{b}=2 \beta T_{c}$ is the DCSK bit frame time interval for each bit. The discrete form of the baseband signal sequence corresponding to the $i^{t h}$ bit of user $l$ at the output of the transmitter denoted by $e_{k, i, l}$ is given by

$$
e_{k, i, l}=\left\{\begin{array}{ll}
x_{k, i} & \text { for }(1<k \leq \beta) \\
s_{i, l} x_{k-\beta, i} & \text { for }(\beta<k \leq 2 \beta)
\end{array},\right.
$$

where $s_{i, l}$ is the $i^{t h}$ bit of user $l$ and $x_{k}$ is the chaotic discrete sequence used as the reference signal and $x_{k-\beta}$ is the delayed version of it.

The block diagram of the general structure of DCSK communication system is depicted in Fig. 1. According to this figure, in order to demodulate the transmitted bits, the received signal $r_{k, i, l}$ is correlated to its delayed version $r_{k+\beta, i, l}$ and summed over the bit duration $T_{b}$ where $T_{b}=\beta T_{c}$ and $T_{c}$ is the chip time. The received bits are then estimated by computing the sign of the correlator output, i.e. see Fig. 1(c).

It can also be inferred from the figure that half the bit frame is dedicated to sending the non-information-bearing reference, and as a consequence, results in the dissipation of half of the bit energy by the reference sequence which can be interpreted as energy inefficiency of the system. Therefore, the data rate of this system is considerably reduced. 


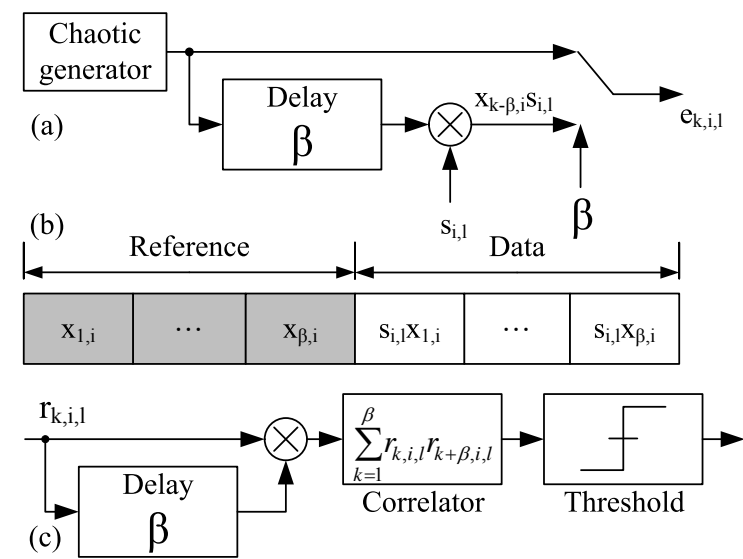

Fig. 1: Block diagram of the general structure of the DCSK communication system for the user $l$; (a) The DCSK transmitter, (b) The DCSK frame, and (c) The DCSK receiver.

\section{B. Channel Model}

This work uses the general channel model of spread spectrum wireless communication systems [16], [41]-[43]. As shown in Fig. 2, a multipath fading channel with $V$ independent paths is considered for each user. The channel coefficient and the time delay of the $v^{\text {th }}$ path for the user node $l$ are expressed by $\lambda_{i, u, l, v}$, and $\tau_{u, l, v}$, respectively. Although the channel coefficient of a given user $l$ is constant during the time of the multiple access and the broadcast phases, it is different for each bit, each data stream $u$, or path $v$. It is essential to note that the index $u$ is not applicable to the ANC-based DCSK scenario and used in the analysis of the ANC-based MC-DCSK to represent the parallel data stream.

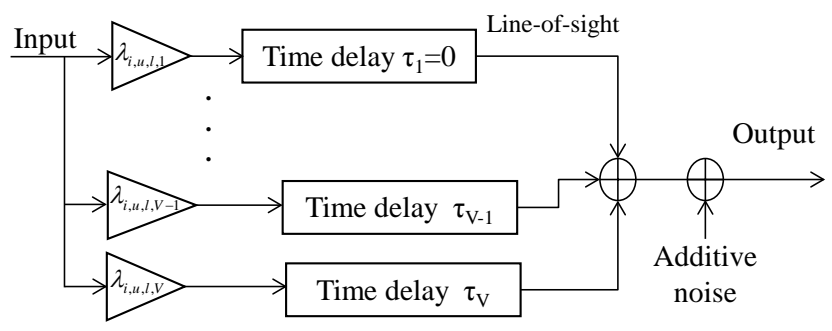

Fig. 2: Multipath fading channel model.

In this study, the channel coefficient $\lambda$ follows a Rayleigh or Rician distributions given in [41], [44]. It should also be underlined that in an AWGN scenario, the number of paths is equal one, and $V=1$ leads to a unit channel coefficient $\lambda_{i, u, l, 1}=1$. The channel coefficients are assumed to be constant during transmission (i.e. during the two times slots) whereas the gain varies over the total bandwidth from one user to another.

Assuming that the delay spread of the channel is much smaller than the symbol duration, the inter-symbol interference can be neglected. Therefore, the proposed model shown in Fig. 2 is valid for Rician multipath channel [43], [45]. Furthermore, the average values of channel coefficients are considered identical for both uplink and downlink channels.
Since the system uses time division multiplexing technique (i.e. multiple access and broadcast phases are separated in time), the channel can be realistically considered symmetric [46]. Therefore, this work considers a symmetric model for the two-way communication channel for each user.

Based on the developed channel estimation algorithms for UWB systems in [7], the exact information of channel state is assumed to be available at the receiver side.

\section{Analysis of the ANC-based DCSK Scheme}

One major advantage of ANC scheme over the Straightforward Network Coding scheme (SNC) is that the number of the required time slots for the communication between two user nodes is reduced from three to two [5]. However, applying the ANC scheme to the conventional DCSK system results in substantial constraints and drawbacks. For instance, the conventional ANC-based DCSK system can not supports more than two users due to the absence of multiple access protocol. Additionally, the general ANC-based DCSK suffers from high interference level that is resulted from the cross multiplication of the user signals.

In the general ANC-based DCSK scheme, a pair of users $A$ and $B$ intending to communicate with each other must use the same spreading code $x$ which allows for exploiting the resultant interference in a way that the two users can still extract the information from the other member of the pair [2]. Table I summarizes the ANC-based DCSK mapping scheme where $s_{A}$ and $s_{B}$ denote the data messages of user nodes $A$ and $B$, while $e_{A}(t), e_{B}(t)$, and $T(t)$ refer to the transmitted signals from the user pair to the relay node $\mathcal{R}$, and from the relay node to the two users, respectively. The transmitted signal represented by $\left[s_{l} x, x\right]$ designates the DCSK frame in which the first term represents the symbol $s_{i, l}=\{-1,+1\}$ multiplied by the reference sequence $x$ and the second term represents the reference sequence $x$. In addition, $s_{D}$ represents the decoded symbols of the received superimposed signal at the user side. As detailed in table I, for instance, from the user $\mathcal{A}$ point of view, after despreading and decoding the received signal, the user node $A$ extracts its own data from the decoded signal to recover the data originating from node $B$.

TABLE I: ANC-based DCSK mapping scheme

\begin{tabular}{|r|r|r|c|c|}
\hline \hline$e_{A}(t)$ & \multicolumn{1}{|c|}{$e_{B}(t)$} & $T(t)$ & $s_{D}$ & $\hat{s}_{B}=s_{D}-s_{A}$ \\
\hline \hline$[1 x, x]$ & {$[1 x, x]$} & {$[2 x, 2 x]$} & 2 & $2-1=1$ \\
\hline$[1 x, x]$ & {$[-1 x, x]$} & {$[0 x, 2 x]$} & 0 & $0-(-1)=1$ \\
\hline$[-1 x, x]$ & {$[1 x, x]$} & {$[0 x, 2 x]$} & 0 & $0-1=-1$ \\
\hline$[-1 x, x]$ & {$[-1 x, x]$} & {$[-2 x, 2 x]$} & -2 & $-2-(-1)=-1$ \\
\hline
\end{tabular}

In this scenario, the two users transmit their signals to the relay in the first time slot. The multi-user signals corresponding to the $i^{\text {th }}$ bit that is received by the relay can be expressed by

$$
T_{i}(t)=\sum_{l=1}^{2} \sum_{v=1}^{V} \lambda_{i, l, v} e_{i, l}\left(t-\tau_{l, v}\right)+w_{i}(t),
$$

where $\lambda_{i, l, v}(t)$ and $\tau_{l, v}$ represent the channel coefficient and the corresponding delay respectively, whereas $w_{i}(t)$ denotes 
the AWGN with zero mean and power spectral density of $N_{0} / 2$.

In the second time slot, the relay forwards the received signal $T(t)$ to the two users in the network. The received signal at the user $A$ side corresponding to the $i^{\text {th }}$ bit can be given as the following

$$
r_{i, A}(t)=\sum_{v^{\prime}=1}^{V} \lambda_{i, 1, v^{\prime}} T\left(t-\tau_{1, v^{\prime}}\right)+n_{i}(t)
$$

Considering the user end $A$, the information transmitted by user $B$ is extracted by despreading, decoding and demapping the received signal. The despreading process at user $A$ is performed by correlating $r_{i, A}$ with its delayed version $r_{i, A}(t+\beta)$ and summing the resultant over a half bit frame time interval $T_{b}$, where $T_{b}=\beta T_{c}$. The decoding and demapping processes are also given in detail in table I.

In this respect, the discreet baseband output of the correlator for users $A$ and $B$ in the ANC-based DCSK scheme is represented by

$$
D_{i, A, B}=\sum_{k=1}^{\beta} r_{k, i, A} r_{k+\beta, i, A}
$$

Using equation (3) in equation (4), the decision variable might be determined by

$$
\begin{gathered}
D_{i, A, B}= \\
\sum_{k=1}^{\beta}\left(\sum_{v,=1}^{V} \lambda_{i, l, v},\left(\sum_{v=1}^{V} \sum_{l=1}^{2} \lambda_{i, l, v} x_{k-\tau_{l, v}-\tau_{l, v},}\right.\right. \\
\left.\left.+w_{k-\tau_{l, v}, i}\right)+n_{k, i}\right) \\
\sum_{k=1}^{\beta}\left(\sum_{v,=1}^{V} \lambda_{i, l, v},\left(\sum_{v=1}^{V} \sum_{l=1}^{2} \lambda_{i, l, v} s_{i, l} x_{k-\tau_{l, v}-\tau_{l, v},}\right.\right. \\
\left.\left.+w_{k+\beta-\tau_{l, v}, i}\right)+n_{k+\beta, i}\right) .
\end{gathered}
$$

As shown in Fig. 2, in presence of line of sight $\left(\tau_{l, 1}=0\right)$, and thus equation (5) can be developed as equation (6).

The first line in equation (6) represents the useful signal while the other components represent the intersymbol interference (ISI), multipath, and the Gaussian noise interferences. As can be seen there are many interference components in the decision variable of the conventional ANC-based DCSK which degrades the system performance substantially. These terms originate from the cross product of the user data signals and the reference ones. Therefore, one can conclude that the general form of the ANC-based DCSK scheme without any interference mitigation technique can not be considered as a preferred candidate for an ANC application.

One way to circumvent the impairment by interference, is to separate the user signals in time or frequency domain. In this respect, the proposed ANC-based MC-DCSK in the next section addresses the interference problem by applying the frequency domain multiplexing technique to separate the data carrier and the reference signal transmitted by different users. This scheme not only mitigates the interference but also allows for the communication of more than two users in the network.

\section{ANC-Based Multi-User Multi-CARrier DCSK COMMUNICATION SYSTEMS}

This section studies the multi-user MC-DCSK system design and shows how its performance is enhanced by the application of the multi-user ANC scheme.

\section{A. Multi-User MC-DCSK System Architecture}

One of the main advantages of the proposed MC-DCSK system in [39] is its high spectral efficiency. Fig. 3 depicts the block diagram of the MC-DCSK system. As can be seen from the figure, the input information sequence for user $l$ is first converted into $U$ parallel independent data sequences where $s_{i, u, l}=\{-1,+1\}$ is the $i^{t h}$ bit of the $u^{t h}$ data sequence of user $l$.

In this scheme, the chaotic signal $x_{u, l}(t)$ used as the reference and the spreading code of a user $l$ can be obtained by

$$
x_{u, l}(t)=\sum_{k=1}^{\beta} x_{k, u, l} h\left(t-k T_{c}\right),
$$

where $\beta$ is the spreading factor, and $h(t)$ is the band-limited square-root-raised-cosine filter which is normalized to have unit energy. After a serial-to-parallel conversion, the $M$-bit stream of the $u^{t h}$ data stream (i.e. $M$ denotes the number of bits per sequence) are spread due to the multiplication by the same chaotic spreading code $x_{u, l}(t)$ in the time domain. It is essential to note that the chaotic reference does not vary for each of $M$ bits but changes for every $u^{t h}$ data sequence.

Let $H(f)=F\{h(t)\}$, where $F$ denotes the Fourier transform function. If $H(f)$ is limited to $\left[-B_{c} / 2, B_{c} / 2\right]$, the Nyquist criterion is satisfied with a roll-off factor $\alpha(0 \leq \alpha \leq$ $1)$, where $B_{c}=(1+\alpha) / T_{c}$. To have a bandwidth-efficient scheme, each user has a predefined subcarrier $f_{l+M}$, allocated to transmitting the reference symbols as well as $M$ subcarriers $f_{i}$, shared with the $L-1$ users to transmit the data symbols. Therefore, the transmitted signal for the $u^{t h}$ data stream of the $l^{t h}$ user in the MC-DCSK system can be given as the following

$$
\begin{aligned}
e_{u, l}(t)= & x_{u, l}(t) \cos \left(2 \pi f_{l+M} t+\phi_{l+M}\right) \\
& +\sum_{i=1}^{M} s_{i, u, l}(t) x_{u, l}(t) \cos \left(2 \pi f_{i} t+\phi_{i}\right),
\end{aligned}
$$

where $\phi_{i}$ represents the phase angle defined in the carrier modulation process.

In this work, the transmitted energy is normalized in each subcarrier. Furthermore, the modulated subcarriers are orthogonal over the chip duration. The baseband frequency corresponding to the $i^{\text {th }}$ subcarrier can be represented by $f_{i}=f_{p}+i \Delta$, where $f_{p}$ is the fundamental subcarrier frequency. Therefore, the minimum frequency spacing between two adjacent subcarriers is equal to $\Delta=(1+\alpha) / T_{c}$, which is one of the commonly used assumptions in similar scenarios [47].

As per the study of energy efficiency in the MC-DCSK system in [39], the reference energy $\mathcal{E}_{\text {ref }}$ can be defined as the energy needed to transmit the reference signal of $M$ bits, while the required energy to transmit one bit is the sum of 


$$
\begin{aligned}
& D_{i, A, B}=\sum_{k=1}^{\beta} \sum_{l=1}^{2} s_{i, l} \lambda_{i, l, 1}^{2} x_{k-2 \tau l, 1}^{2} \\
& +\left(\sum_{k=1}^{\beta} \sum_{v,=2}^{V} \sum_{v=2}^{V} \sum_{l=1}^{2} \lambda_{i, l, v} \lambda_{i, l, v}, x_{k-\tau_{l, v}-\tau_{l, v}}\right)\left(\sum_{k=1}^{\beta} \sum_{v,=2}^{V} \sum_{v=2}^{V} \sum_{l=1}^{2} \lambda_{i, l, v} \lambda_{i, l, v}, s_{i, l} x_{k-\tau_{l, v}-\tau_{l, v},}\right) \\
& +\left(\sum_{k=1}^{\beta} \sum_{v,=1}^{V} \sum_{v=1}^{V} \sum_{l=1}^{2} \lambda_{i, l, v} \lambda_{i, l, v}, x_{k-\tau_{l, v}-\tau_{l, v}}\right)\left(\sum_{k=1}^{\beta} \sum_{v,=1}^{V} \lambda_{i, l, v}, w_{k+\beta-\tau_{l, v}, i}\right)+\left(\sum_{k=1}^{\beta} n_{k, i} n_{k+\beta, i}\right) \\
& +\left(\sum_{k=1}^{\beta} \sum_{v,=2}^{V} \sum_{v=2}^{V} \sum_{l=1}^{2} \lambda_{i, l, v} \lambda_{i, l, v}, x_{k-\tau_{l, v}-\tau_{l, v}}, n_{k+\beta, i}\right)+\left(\sum_{k=1}^{\beta} \sum_{v,=2}^{V} \sum_{v=2}^{V} \sum_{l=1}^{2} \lambda_{i, l, v} \lambda_{i, l, v}, s_{i, l} x_{k-\tau_{l, v}-\tau_{l, v}, n_{k, i}}\right) \\
& +\left(\sum_{k=1}^{\beta} \sum_{v,=1}^{V} \sum_{v=1}^{V} \sum_{l=1}^{2} \lambda_{i, l, v} \lambda_{i, l, v}, s_{i, l} x_{k-\tau_{l, v}-\tau_{l, v}}\right)\left(\sum_{k=1}^{\beta} \sum_{v,=1}^{V} \lambda_{i, l, v}, w_{k-\tau_{l, v}, i}\right)+\left(\sum_{k=1}^{\beta} \sum_{v,=1}^{V} \lambda_{i, l, v}, w_{k-\tau_{l, v}, i} n_{k+\beta, i}\right) \\
& +\left(\sum_{k=1}^{\beta} \sum_{v,=1}^{V} \lambda_{i, l, v}, w_{k+\beta-\tau_{l, v}, i} n_{k, i}\right)+\left(\sum_{k=1}^{\beta} \sum_{v,=1}^{V} \lambda_{i, l, v}, w_{k+\beta-\tau_{l, v}, i}\right)\left(\sum_{k=1}^{\beta} \sum_{v,=1}^{V} \lambda_{i, l, v}, w_{k+\beta}-\tau_{l, v}, i\right)
\end{aligned}
$$

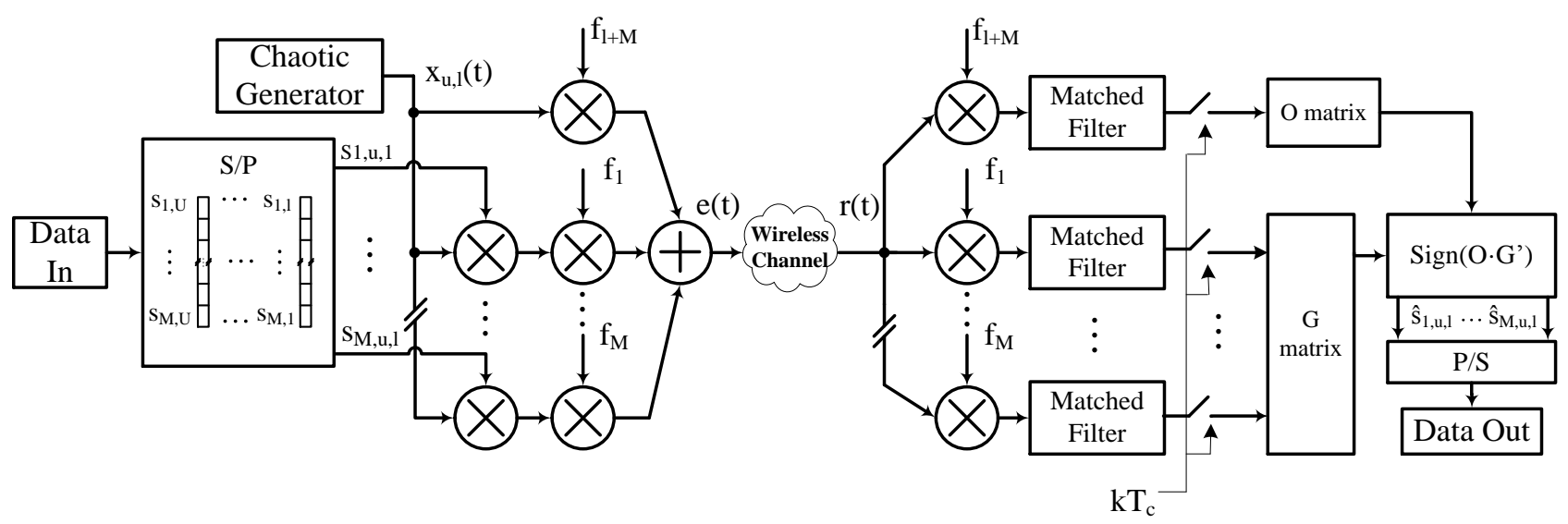

Fig. 3: Block diagram of the MC-DCSK system for the user $l$.

its data carrier energy $\mathcal{E}_{\text {data }}$. The required reference energy for transmitting each bit can be obtained as the following

$$
\mathcal{E}_{b}=\mathcal{E}_{\text {data }}+\frac{\mathcal{E}_{\text {ref }}}{M}
$$

and the energies of the $M+1$ subcarriers can be defined as

$$
\mathcal{E}_{\text {data }}=\mathcal{E}_{\text {ref }}=T_{c} \sum_{k=1}^{\beta} x_{k, i}^{2} .
$$

Therefore, the bit energy expression as a function of $E_{\text {data }}$ can be given as the following

$$
\mathcal{E}_{b}=\frac{M+1}{M} \mathcal{E}_{\text {data }} .
$$

According to Fig. 3, after removing the carrier frequencies and sampling the corresponding signals in every $T_{c}$, the discrete signals at the output are stored in a matrix memory. The matrix implementation simplifies data recovery by performing the decoding process in a parallel scheme.

In the proposed multi-user communication scheme, each user node is assigned a private frequency to transmit the reference signal, while the rest of the available bandwidth is shared among all users for data symbol transmissions. Fig. 4 demonstrates the power spectral density (PSD) of the multiuser MC-DCSK system. This informative figure shows that the reference signals are separated in the frequency domain, and that the data signals of the $L$ nodes are spread by different chaotic spreading codes over the remaining shared bandwidth.

The decoding algorithm in a mono-user scenario under an AWGN channel can be described in two parts:

1). The reference and the data signals are simultaneously stored in the $1 \times \beta$ matrix $\mathbf{O}$ and $M \times \beta$ matrix $\mathbf{G}$, respectively. Therefore $\mathbf{O}$ can be given as the following

$$
\mathbf{O}=\left(x_{1, u, l}+n_{1, u, l}, x_{2, u, l}+n_{2, u, l}, \ldots x_{\beta, u, l}+n_{\beta, u, l}\right),
$$

where $n_{k, u, l}$ is the $k^{t h}$ sample of additive Gaussian noise that is added to the reference signal generated for the $u^{t h}$ data stream of user $l$. Therefore, the matrix $\mathbf{G}$ is given by

$\mathbf{G}=\left(\begin{array}{ccc}s_{1, u, l} x_{1, u, l}+n_{1,1, u, l} & \ldots & s_{1, u, l} x_{\beta, u, l}+n_{\beta, 1, u, l} \\ \vdots & \vdots & \vdots \\ s_{M, u, l} x_{1, u, l}+n_{1, M, u, l} & \ldots & s_{M, u, l} x_{\beta, u, l}+n_{\beta, M, u, l}\end{array}\right)$,

where $n_{k, i, u, l}$ is the $k^{t h}$ sample of additive Gaussian noise added to the $i^{t h}$ bit of $u^{\text {th }}$ data stream of user $l$.

2). After $\beta$ clock cycles when all of the samples are stored, the decoding process is carried out by the destination node. In 


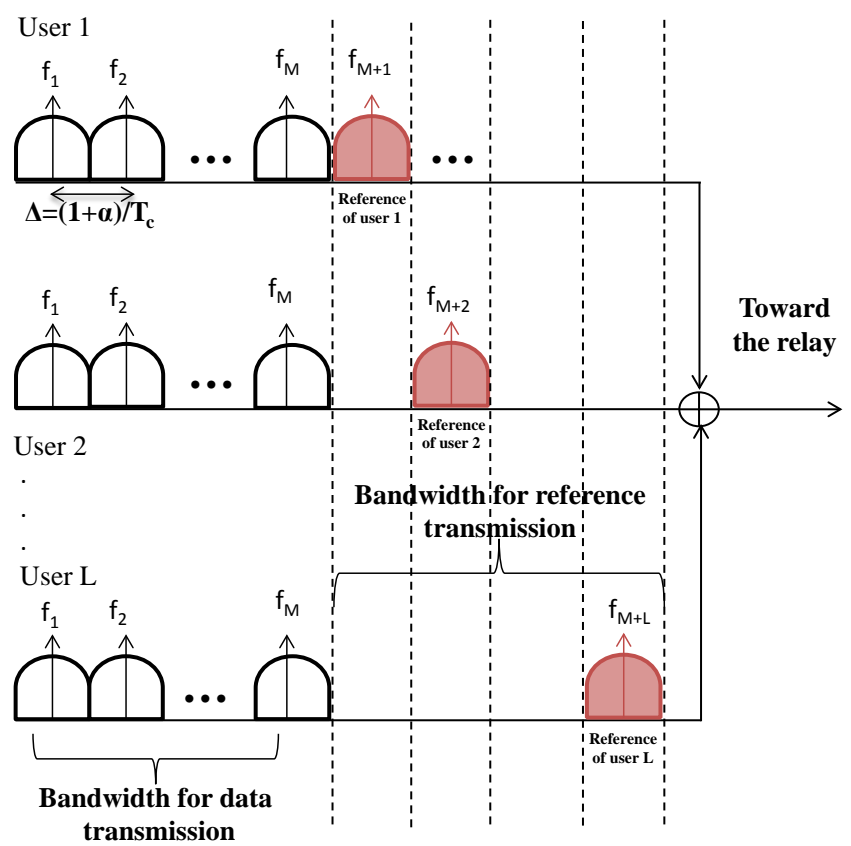

Fig. 4: The power spectral density of a band-limited multi-user MC-DCSK system.

this process, the transmitted $M$ bits are recovered, in parallel, by computing the sign of the resultant vector from the matrix product given as follow

$$
\hat{\mathbf{s}}_{\mathbf{u}}=\operatorname{sign}\left(\mathbf{O} \times \mathbf{G}^{\prime}\right),
$$

where $x$ is the matrix product, ' represents the transpose operator, and $\hat{\mathbf{s u}^{\prime}}=\left\{s_{i, u}, \ldots, s_{M-1, u}, s_{M, u}\right\}$ denotes the transmitted bits of the $u^{t h}$ data stream. In fact, this matrix product is equivalent to the output of a set of parallel correlators where the reference signal is multiplied by each data slot, and the result is summed over $\beta T c$.

\section{B. Analog Network Coding Scheme}

This paper presents a new design of ANC-based MC-DCSK modulation scheme and evaluates its performance bound over multipath fading channels. Fig. 5 shows the proposed system topology, where all $L$ user nodes can communicate with each other through a relay node $R$. Furthermore, this topology enables the nodes 1 to $L$ to transmit their data simultaneously, by exploiting the network coding operation that is performed on the superimposed electromagnetic waves. Additionally, thanks to the non-coherent detection, each user can decode the received signal from the other users, by knowing the frequency assigned for transmitting the reference signal. Finally, the relay forwards the sum of signals without any amplification. However, the amplification process can be simply added to such a system if it is needed, as the relay has sufficient resources to carry out this operation.

\section{Multiple Access Phase}

In the first time slot, all users transmit their signals to the relay node. The multi-user signals corresponding to the $i^{\text {th }}$ bit

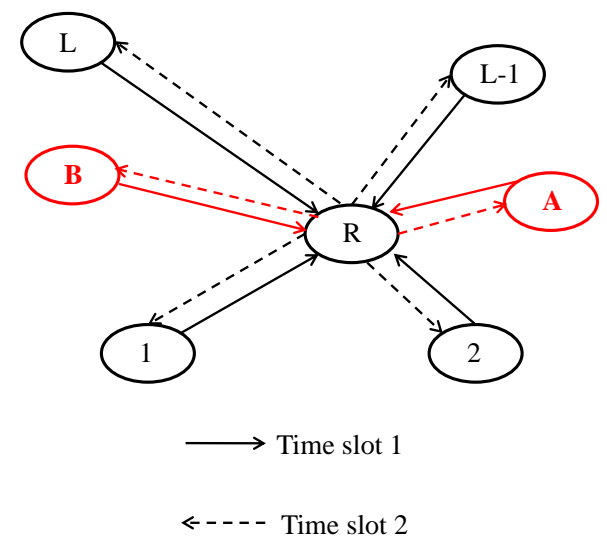

Fig. 5: ANC-based MC-DCSK network topology, while users $A$ and $B$ are communicating through the relay node $R$.

of the $u^{\text {th }}$ data stream that is received by the relay can be expressed by

$$
T_{i, u}(t)=\sum_{l=1}^{L} \sum_{v=1}^{V} \lambda_{i, u, l, v} e_{l}\left(t-\tau_{u, l, v}\right)+w_{i, u}(t),
$$

where $\lambda_{i, u, l, v}(t)$ and $\tau_{u, l, v}$ represent the channel coefficient and the corresponding delay, respectively. Furthermore, $L$ and $w_{i, u}(t)$ denote the number of users (i.e. here $L \geq 2$ ), and AWGN with zero mean and power spectral density of $N_{0} / 2$, in the named order.

\section{Broadcast Phase}

In the second time slot, the relay simply broadcasts its received signal $T(t)$. The received signal at the $A^{t h}$ user end corresponding to the $i^{t h}$ bit of the $u^{t h}$ data stream can be obtained as the following

$$
r_{i, u, A}(t)=\sum_{v^{\prime}=1}^{V} \lambda_{i, u, A, v^{\prime}} T\left(t-\tau_{u, A, v^{\prime}}\right)+n_{i, u}(t),
$$

where $\lambda_{i, u, A, v^{\prime}}(t)$ represents the $v^{\text {th }}$ downlink channel path coefficient between the relay and user $A$, and $n_{i, u}(t)$ denotes an AWGN with zero mean with power spectral density of $N_{0} / 2$.

Since the channel is symmetric, by developing the received signal and isolating for the signal components of user $A$, the resulting signal corresponding to the $i^{\text {th }}$ bit of the $u^{\text {th }}$ data stream can be expressed by

$$
\begin{aligned}
& r_{i, u, A}(t)=\sum_{v=1}^{V} \lambda_{i, u, A, v}^{2} e_{i, u, A}\left(t-2 \tau_{u, A, v}\right) \\
& +\sum_{v=1}^{V} \sum_{\substack{v^{\prime}=1 \\
v^{\prime} \neq v}}^{V} \lambda_{i, u, A, v} \lambda_{i, u, A, v}, e_{i, u, A}\left(t-\tau_{u, A, v}-\tau_{u, A, v^{\prime}}\right) \\
& +\sum_{\substack{l=1 \\
l \neq A}}^{V} \sum_{v=1}^{V} \sum_{v^{\prime}=1}^{V} \lambda_{i, u, A, v^{\prime}} \lambda_{i, u, l, v} e_{l}\left(t-\tau_{u, l, v}-\tau_{u, A, v^{\prime}}\right) \\
& +\sum_{v^{\prime}=1}^{V} \lambda_{i, u, A, v^{\prime}} w_{i, u}\left(t-\tau_{u, A, v}\right)+n_{i, u}(t) .
\end{aligned}
$$




\section{E. Removing Self-Interference and Decoding}

Fig. 6 summarizes the block diagram of the proposed ANCbased MC-DCSK form the point of view of the user $A$ as a reviver while decoding the transmitted data of user $B$. In the second time slot, the received signal $r_{i, u, A}(t)$ at user $A$ side (after removing the carrier frequencies) consists of two distinct signal components, the data signal $q_{i, u, A}(t)$, and the reference signal $\rho_{u, B}(t)$. The former comprises the spread version of $M$ data signals transmitted by the $L$ users and can be recovered by using the common frequencies $\left\{f_{1}, \ldots f_{M-1}, f_{M}\right\}$, whereas the latter is the reference signal of user $B$ transmitted over the predefined frequency $f_{B+M}$ to user $A$.

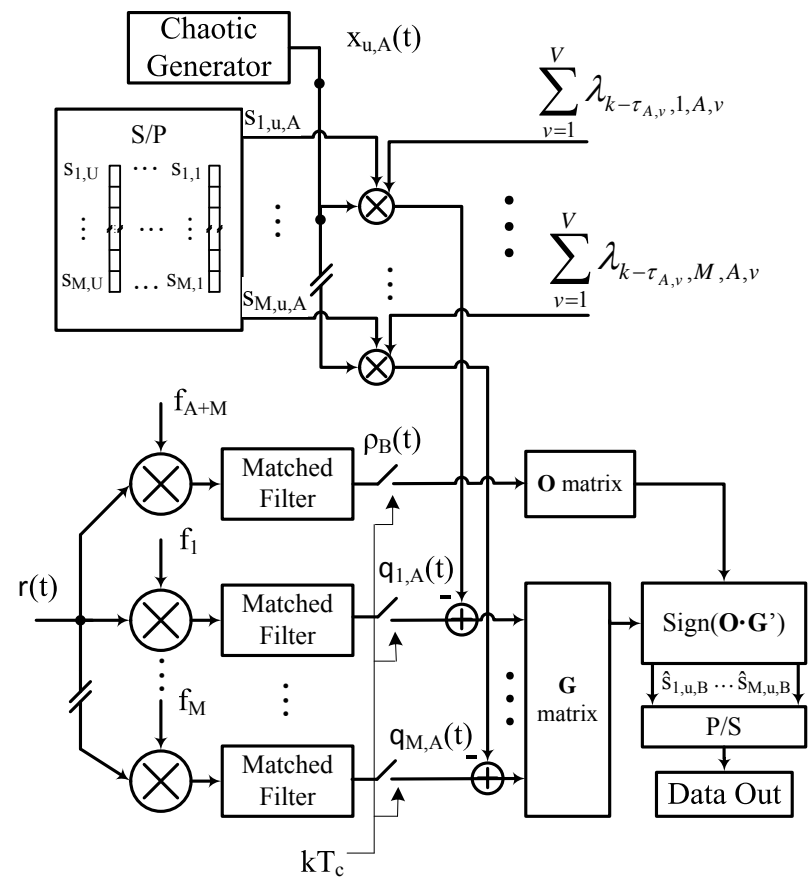

Fig. 6: Block diagram of the receiver $A$ that decodes the signal transmitted by user $B$, in the ANC-based MC-DCSK system.

In order to eliminate the self-interference from the signal $q_{i, u, A}(t)$ at the receiver $A$, it is required to determine the channel state information of user $A$. The channel state information of user $A$ is assumed to be perfectly estimated. According to Fig. 6, the local reference signal is multiplied by the corresponding bit and the channel coefficients. The resulting signal is then subtracted from the signal $q_{i, u, A}(t)$ to remove the data signal of user $A$.

The multi-user data information signal, $q_{i, u, A}(t)$, corresponding to the $u^{t h}$ data stream, for the the $i^{\text {th }}$ bit of user
$A$ after self-interference cancellation can be determined by

$$
\begin{aligned}
& q_{i, u, A}(t)=\sum_{v=1}^{V} \lambda_{i, u, A, v}^{2} s_{i, u, A} x_{A}\left(t-2 \tau_{u, A, v}\right) \\
& +\sum_{v=1}^{V} \sum_{\substack{v^{\prime}=1 \\
v^{\prime} \neq v}}^{V} \lambda_{i, u, A, v} \lambda_{i, u, A, v^{\prime}} s_{i, u, A} x_{A}\left(t-\tau_{u, A, v}-\tau_{u, A, v^{\prime}}\right) \\
& +\sum_{\substack{l=1 \\
l \neq A}}^{L} \sum_{v=1}^{V} \sum_{v^{\prime}=1}^{V} \lambda_{i, u, A, v^{\prime}} \lambda_{i, u, l, v} s_{i, u, l} x_{l}\left(t-\tau_{u, l, v}-\tau_{u, A, v^{\prime}}\right) \\
& +\sum_{v^{\prime}=1}^{V} \lambda_{i, u, A, v^{\prime}} w_{i, u}\left(t-\tau_{u, A, v^{\prime}}\right)+n_{i, u}(t) \\
& -\sum_{v=1}^{V} \lambda_{i, u, A, v}^{2} s_{i, u, A} x_{A}\left(t-2 \tau_{u, A, v}\right) \\
& -\sum_{v=1}^{V} \sum_{\substack{v^{\prime}=1 \\
v^{\prime} \neq v}}^{V} \lambda_{i, u, A, v} \lambda_{i, u, A, v^{\prime}} s_{i, u, A} x_{A}\left(t-\tau_{u, A, v}-\tau_{u, A, v^{\prime}}\right),
\end{aligned}
$$

where the first and the second terms of this equation is attributed to the self-interference of the user $A$. Therefore, the multi-user data signal in (18) can be simplified to

$$
\begin{aligned}
& q_{i, u, A}(t)= \\
& \sum_{\substack{l=1 \\
l \neq A}}^{L} \sum_{v=1}^{V} \sum_{v^{\prime}=1}^{V} \lambda_{i, u, A, v^{\prime}} \lambda_{i, u, l, v} s_{i, u, l} x_{l}\left(t-\tau_{u, l, v}-\tau_{u, A, v^{\prime}}\right) \\
& +\sum_{v^{\prime}=1}^{V} \lambda_{i, u, A, v^{\prime}} w_{i, u}\left(t-\tau_{u, A, v^{\prime}}\right)+n_{i, u}(t)
\end{aligned}
$$

It should be noted that the noise signals $\left(w_{i, u}(t)\right.$ and $\left.n_{i, u}(t)\right)$ of the $i^{t h}$ bit transmitted over the frequency $f_{i}$, are added to the signal $q_{i, u, A}(t)$.

To decode the $i^{t h}$ bit of the $u^{t h}$ stream corresponding to user $B$, user $A$ recovers the reference signal (the spreading sequence) that is transmitted by user $B$ over the frequency $f_{M+B}$. In this respect, the received chaotic signal or the reference signal related to the $u^{t h}$ data stream of user $B$ can be obtained by

$$
\begin{aligned}
\rho_{u, B}(t)= & \sum_{v=1}^{V} \sum_{v^{\prime}=1}^{V} \lambda_{u, \Re_{A}, v^{\prime}} \lambda_{u, \Re_{B}, v} x_{B}\left(t-\tau_{u, B, v}-\tau_{u, A, v^{\prime}}\right) \\
& +\sum_{v^{\prime}=1}^{V} \lambda_{u, \Re_{A}, v^{\prime}} w_{u, B}\left(t-\tau_{A, v^{\prime}}\right)+n_{u, B}(t),
\end{aligned}
$$

where $w_{u, B}(t)$ and $n_{u, B}(t)$ are the noise signals that are added to the signal $\rho_{u, B}(t)$ before being transmitted over the frequency $f_{B+M}$. The channel coefficient of the $v^{t h}$ path expressed by $\lambda_{u, \Re_{l}, v}$, is then multiplied by the reference signal of the user $l$ corresponding to the $u^{t h}$ data stream.

Once the self-interference of user $A$ is removed from the data signal $q_{i, u, A}(t)$ and the reference sequence $\rho_{u, B}(t)$ of user $B$ is recovered, the channel compensation should be performed by using the obtained $\lambda_{i, u, A, v}$ and $\lambda_{i, u, B, v}$. Therefore, the 
multi-user data signal can be determined by

$$
\begin{aligned}
& q_{i, u, A}(t)= \\
& \sum_{\substack{l=1 \\
l \neq 1}}^{L} \sum_{v=1}^{V} \sum_{v^{\prime}=1}^{V} \lambda_{i, u, l, v} \lambda_{i, u, B, v} \lambda_{i, u, A, v^{\prime}}^{2} s_{i, u, l} x_{l}\left(t-\tau_{u, l, v}-\tau_{u, A, v^{\prime}}\right) \\
& +\sum_{v=1}^{V} \lambda_{i, u, A, v}^{2} \lambda_{i, u, B, v} w_{i, u}\left(t-\tau_{u, A, v}\right) \\
& +\sum_{v=1}^{V} \lambda_{i, u, A, v} \lambda_{i, u, B, v} n_{i, u}\left(t-\tau_{u, A, v}\right) .
\end{aligned}
$$

Similarly, the reference chaotic signal of user $B$ that is recovered by user $A$ is

$$
\begin{aligned}
& \rho_{u, B}(t)= \\
& \sum_{v=1}^{V} \sum_{v^{\prime}=1}^{V} \lambda_{u, \Re_{A}, v^{\prime}}^{2} \lambda_{u, \Re_{B}, v}^{2} x_{B}\left(t-\tau_{u, B, v}-\tau_{u, A, v^{\prime}}\right) \\
& +\sum_{v=1}^{V} \lambda_{u, \Re_{A}, v}^{2} \lambda_{u, \Re_{B}, v} w_{u, B}\left(t-\tau_{u, A, v}\right) \\
& +\sum_{v=1}^{V} \lambda_{u, \Re_{A}, v} \lambda_{u, \Re_{B}, v} n_{u, B}\left(t-\tau_{u, A, v}\right) .
\end{aligned}
$$

After channel compensation at the output of the correlator, the decision variable of user $A$ for the $i^{t h}$ bit of the $u^{t h}$ data stream corresponding to user $B$ may be given by

$$
D_{i, u, A, B}=\sum_{k=1}^{\beta} \rho_{k, u, B} q_{k, i, u, A} .
$$

Using (21) and (22) in (23) yields

$$
D_{i, u, A, B}=\Psi+Y+W+Z+N
$$

where $\Psi$ denotes the useful signal expressed by

$$
\begin{aligned}
\Psi= & \sum_{k=1}^{\beta} \sum_{v=1}^{V} \sum_{v^{\prime}=1}^{V}\left(\lambda_{u, \Re_{A}, v^{\prime}}^{2} \lambda_{u, \Re_{B}, v}^{2} x_{k-\tau_{u, B, v}-\tau_{u, A, v^{\prime}, B}}^{2}\right) \\
& \left(\lambda_{i, u, A, v^{\prime}}^{2} \lambda_{i, u, B, v}^{2} x_{k-\tau_{u, B, v}-\tau_{u, A, v^{\prime}, B}}^{2} s_{i, u, B}\right)
\end{aligned}
$$

and $Y$ represents multi-user interference which can be given by

$$
\begin{aligned}
Y= & \sum_{k=1}^{\beta} \sum_{\substack{l=1 \\
l \neq A \\
l \neq B}}^{L} \sum_{v=1}^{V} \sum_{v^{\prime}=1}^{V}\left(\lambda_{u, \Re_{A}, v^{\prime}}^{2} \lambda_{u, \Re_{B}, v}^{2} \lambda_{i, u, l, v} \lambda_{i, u, B, v} \lambda_{i, A, v^{\prime}}^{2}\right) \\
& \left(s_{i, u, l} x_{k-\tau_{u, l, v}-\tau_{u, A, v^{\prime}}, A} x_{k-\tau_{u, B, v}-\tau_{u, A, v^{\prime}, B}}\right) \cdot
\end{aligned}
$$

Additionally, $W, Z$, and $N$ given in equation (24) are the noise interference components which can receptively be obtained as the followings

$$
\begin{aligned}
& W=\sum_{k=1}^{\beta} \sum_{v=1}^{V} \sum_{v^{\prime}=1}^{V}\left(\lambda_{u, \Re_{A}, v^{\prime}}^{2} \lambda_{u, \Re_{B}, v}^{2} x_{k-\tau_{u, B, v}-\tau_{u, A, v^{\prime}}, B}\right) \\
& \left(\lambda_{i, u, A, v^{\prime}}^{2} \lambda_{i, u, B, v} w_{k-\tau_{u, A, v^{\prime}, i}}+\lambda_{i, u, A, v^{\prime}} \lambda_{i, u, B, v} n_{k-\tau_{u, A, v^{\prime}, i}}\right),
\end{aligned}
$$

$$
\begin{aligned}
& Z=\sum_{k=1}^{\beta} \sum_{v=1}^{V} \sum_{v^{\prime}=1}^{V} \lambda_{u, \Re_{A}, v^{\prime}}^{2} \lambda_{u, \Re_{B}, v} w_{k-\tau_{u, A, v^{\prime}}, B} \\
& +\left(\left(\lambda_{u, \Re_{A}, v^{\prime}} \lambda_{u, \Re_{B}, v} n_{k-\tau_{u, A, v^{\prime}, B}}\right)\right. \\
& \left(\sum_{\substack{l=1 \\
l \neq=}}^{L} \lambda_{i, u, l, v} \lambda_{i, u, A, v^{\prime}}^{2} \lambda_{i, u, B, v} s_{i, u, l} x_{\left.k-\tau_{u, l, v}-\tau_{u, A, v^{\prime}, l}\right)}\right),
\end{aligned}
$$

and

$$
\begin{aligned}
N= & \sum_{v=1}^{V} \sum_{v^{\prime}=1}^{V}\left(\lambda_{u, \Re_{A}, v^{\prime}}^{2} \lambda_{u, \Re_{B}, v} w_{k-\tau_{u, A, v^{\prime}}, B}\right. \\
& \left.+\lambda_{u, \Re_{A}, v^{\prime}} \lambda_{u, \Re_{B}, v} n_{k-\tau_{u, A, v^{\prime}}, B}\right) \\
& \left(\lambda_{i, u, A, v^{\prime}}^{2} \lambda_{i, u, B, v} w_{k-\tau_{u, A, v^{\prime}, i}}\right. \\
& \left.+\lambda_{i, u, A, v^{\prime}} \lambda_{i, u, B, v} n_{k-\tau_{u, A, v^{\prime}}, i}\right) .
\end{aligned}
$$

The $i^{\text {th }}$ bit of the $u^{\text {th }}$ data stream corresponding to user $B$ can be decoded by user $A$ comparing the output $D_{i, u, A, B}$ to a zero threshold.

The output of the correlator for the MC-DCSK scenario in (24) can be represented by

$$
\begin{aligned}
D_{i, A, B}= & s_{i, B} \frac{M \sum_{v=1}^{V} \sum_{v^{\prime}=1}^{V} \lambda_{u, \Re_{A}, v^{\prime}}^{2} \lambda_{u, \Re_{B}, v}^{2} \lambda_{i, u, A, v^{\prime}}^{2} \lambda_{i, u, B, v}^{2} \mathcal{E}^{(u)}}{M+1} \\
& +Y+W+Z+N,
\end{aligned}
$$

where $Y, W, Z$, and $N$ represent the interference terms listed in (24), while $\mathcal{E}_{b}^{(u)}$ denotes the transmitted bit energy for a given data stream $u$ of the user $B$

$$
\mathcal{E}_{b}^{(u)}=\frac{M+1}{M} \sum_{k=1}^{\beta} x_{k, u}^{2} .
$$

It should be noticed that the condition of $\tau_{u, l, v}<<\beta T c$ is commonly used in many practical applications [48], [42]. Additionally, in [16] and [42], it has been stated that this interference is negligible for $\tau_{u, l, v}<<\beta T_{c}$, and the limits of this assumption has been studied in [39], [16]. Therefore, this paper also assumes that the multipath delay is much shorter than the bit duration, $0<\tau_{u, l, v}<<T_{b}=\beta T_{c}$.

In this respect, since the correlation between different chaotic signals is very low and the multipath delay is much shorter than the bit duration, the interference between different sequences (i.e. multi-user interference) is negligible compared to the interference within each symbol due to multipath delay. However, multi-user interference has a high tendency to increase with the delay $\tau_{u, l, v}$, and thus degrading the BER significantly. Consequently, in case of large spreading factor scenarios we have

$$
\sum_{k=1}^{\beta}\left(x_{k-\tau_{u, v}} x_{k-\tau_{u, v^{\prime}}}\right) \approx 0 \text { for } v \neq v^{\prime},
$$

and based on this assumption, the interference term of $Y$ is negligible $(Y \approx 0)$. It should be noted that all the terms in equation (30) are uncorrelated. The interference terms of $W$ and $Z$ are zero mean, and that the noise samples as well as the channel coefficients are independent.

However, in a scenario with high number of paths, the multipath delay is much longer than the bit duration, and consequently, the inter-symbol interference (ISI) between different sequences becomes considerable to be taken into account. In this case, a new design of MC-DCSK receiver would be needed that is able to mitigate the ISI and takes advantage of the multipath diversity. This receiver can be similar to a RAKE receiver consisting of parallel DCSK demodulators 
which is able to increase the signal to noise ratio by using the appropriate channel delays.

\section{Performance Analysis of A Multi-User ANC-BASED MC-DCSK SYSTEM}

In this section, the performance of the ANC-Based MCDCSK scheme in multi-user transmission scenario is evaluated. Furthermore, the BER expression under an AWGN and multipath fading channels is analytically derived. To this end, the mean and the variance expressions of the decision variable $D_{i, u, A, B}$ must be determined. Basically, the statistical properties of $D_{i, u, A, B}$ are highly dependent on some properties of chaotic signals. Chaotic generators due to their high sensitivity upon initial conditions are able to generate independent chaotic sequences. It has been stated that these chaotic sequences are also independent of Gaussian noise [11].

In this work, the second-order Chebyshev polynomial function (CPF) is employed to generate chaotic sequences due to its easiness and good performance [49]. For simplicity, the chip time is set to one $\left(T_{c}=1\right)$,

$$
x_{k+1}=1-2 x_{k}^{2} \text {. }
$$

The variance of the normalized chaotic map with zero is equal to one $\left(\operatorname{Var}(x)=\mathrm{E}\left[x^{2}\right]=1\right.$, where $\mathrm{E}[$.$] denotes the$ expected value operator). Therefore, for a given $i^{\text {th }}$ bit of an $u^{t h}$ data stream, the instantaneous mean of the decision variable is the mean of the useful signal which can be given by

$$
\begin{aligned}
& \mathrm{E}\left(D_{i, u, A, B}\right)= \\
& s_{i, B} \frac{M \sum_{v=1}^{V} \sum_{v^{\prime}=1}^{V} \lambda_{u, \Re_{A}, v^{\prime}}^{2} \lambda_{u, \Re_{B}, v}^{2} \lambda_{i, u, A, v^{\prime}}^{2} \lambda_{i, u, B, v}^{2} \mathcal{E}_{b}^{(u)}}{M+1} .
\end{aligned}
$$

Hence, the conditional variance of the decision variable for a given $i^{t h}$ bit may be expressed as the following

$$
\begin{aligned}
& \operatorname{Var}\left(D_{i, u, A, B}\right)= \\
& \mathrm{E}\left[\left(s_{i, u, B} \frac{M \sum_{v=1}^{V} \sum_{v^{\prime}=1}^{V} \lambda_{u, \Re_{A}, v^{\prime}}^{2} \lambda_{u, \Re_{B}, v}^{2} \lambda_{i, u, A, v^{\prime}}^{2} \lambda_{i, u, B, v}^{2} \mathcal{E}_{b}^{(u)}}{M+1}\right)^{2}\right] \\
& +\mathrm{E}\left[W^{2}\right]+\mathrm{E}\left[Z^{2}\right]+\mathrm{E}\left[N^{2}\right]-\left(\mathrm{E}\left[D_{i, u, A, B}\right]\right)^{2},
\end{aligned}
$$

It should be mentioned that for a fixed bit ( $i^{\text {th }}$ bit), the first and the last terms are equal and thus cancelling each other out. Therefore, the total variance of the different interference components of the decision variable can be obtained by

$$
\operatorname{Var}\left(D_{i, u, A, B}\right)=\mathrm{E}\left[W^{2}\right]+\mathrm{E}\left[Z^{2}\right]+\mathrm{E}\left[N^{2}\right]
$$

As mentioned earlier, chaotic sequences are uncorrelated and independent from the channel coefficients as well as from the noise signals. Thus, the terms $W$ can be given by

$$
\begin{aligned}
\mathrm{E}\left[W^{2}\right]= & \mathcal{E}_{b}^{(u)} \sum_{v=1}^{V} \sum_{v^{\prime}=1}^{V} \lambda_{u, \Re_{A}, v^{\prime}}^{4} \lambda_{u, \Re_{B}, v}^{4} \\
& \left(\lambda_{i, u, A, v^{\prime}}^{4} \lambda_{i, u, l, v}^{2}+\lambda_{i, u, A, v^{\prime}}^{2} \lambda_{i, u, l, v}^{2}\right) \frac{N_{0}}{2},
\end{aligned}
$$

which yields

$$
\begin{aligned}
\mathrm{E}\left[W^{2}\right]= & \mathcal{E}_{b}^{(u)} \sum_{v=1}^{V} \sum_{v^{\prime}=1}^{V} \lambda_{u, \Re_{A}, v^{\prime}}^{4} \lambda_{u, \Re_{B}, v}^{4} \lambda_{i, u, A, v^{\prime}}^{2} \lambda_{i, u, B, v}^{2} \\
& \left(\lambda_{i, u, A, v^{\prime}}^{2}+1\right) \frac{N_{0}}{2} .
\end{aligned}
$$

When it comes to $Z$, the variance can be obtained by

$$
\begin{aligned}
\mathrm{E}\left[Z^{2}\right]= & \sum_{v=1}^{V} \sum_{v^{\prime}=1}^{V} \mathcal{E}_{b}^{(u)}\left(\lambda_{u, \Re_{A}, v^{\prime}}^{4} \lambda_{u, \Re_{B}, v}^{2}+\lambda_{u, \Re_{A}, v^{\prime}}^{2} \lambda_{u, \Re_{B}, v}^{2}\right) \\
& \frac{N_{0}}{2} \lambda_{i, u, A, v^{\prime}}^{4} \lambda_{i, u, B, v}^{2} \sum_{\substack{l=1 \\
l \neq A}}^{L} \lambda_{i, u, l, v}^{2},
\end{aligned}
$$

which can be simplified to

$$
\begin{aligned}
\mathrm{E}\left[Z^{2}\right]= & \sum_{v=1}^{V} \sum_{v^{\prime}=1}^{V} \mathcal{E}_{b}^{(u)} \lambda_{u, \Re_{A}, v^{\prime}}^{2} \lambda_{u, \Re_{B}, v}^{2} \lambda_{i, u, A, v^{\prime}}^{4} \lambda_{i, u, B, v}^{4} \\
& \left(\lambda_{u, \Re_{A}, v^{\prime}}^{2}+1\right) \frac{N_{0}}{2} \sum_{\substack{l=1 \\
l \neq A \\
l \neq B}}^{L} \lambda_{i, u, l, v}^{2} .
\end{aligned}
$$

Similarly, the variance of the term $N$ can be expressed by

$$
\begin{aligned}
\mathrm{E}\left[N^{2}\right]= & \sum_{v=1}^{V} \sum_{v^{\prime}=1}^{V} \frac{N_{0}^{2}}{4}\left(\lambda_{u, \Re_{A}, v^{\prime}}^{4} \lambda_{u, \Re_{B}, v}^{2}+\lambda_{u, \Re_{A}, v^{\prime}}^{2} \lambda_{u, \Re_{B}, v}^{2}\right) \\
& \left(\lambda_{i, u, A, v^{\prime}}^{4} \lambda_{i, u, B, v}^{2}+\lambda_{i, u, A, v^{\prime}}^{2} \lambda_{i, u, B, v}^{2}\right)
\end{aligned}
$$

which is simplified to

$$
\begin{aligned}
\mathrm{E}\left[N^{2}\right]= & \sum_{v=1}^{V} \sum_{v^{\prime}=1}^{V} \frac{N_{0}^{2}}{4} \lambda_{u, \Re_{A}, v^{\prime}}^{2} \lambda_{u, \Re_{B}, v}^{2} \lambda_{i, u, A, v^{\prime}}^{2} \lambda_{i, u, B, v}^{2} \\
& \left(\lambda_{u, \Re_{A}, v^{\prime}}^{2}+1\right)\left(\lambda_{i, u, A, v^{\prime}}^{2}+1\right) .
\end{aligned}
$$

In order to analyze the performance of the system, for a given received energy $\mathcal{E}_{b}^{(u)}$, the channel coefficients and the number of paths $V$, the bit error probability (BER) must be determined. Since the bit energy (or chaotic chips) is a deterministic variable, the decision variable at the output of the correlator is necessarily a random Gaussian variable. Therefore, the bit error probability is represented by

$$
\begin{aligned}
\mathrm{BER}= & \frac{1}{2} \operatorname{Pr}\left(D_{i, u, A, B}<0 \mid s_{i, u, B}=+1\right) \\
& +\frac{1}{2} \operatorname{Pr}\left(D_{i, u, A, B}>0 \mid s_{i, u, B}=-1\right),
\end{aligned}
$$

which can be given as follow

$$
\mathrm{BER}=\frac{1}{2} \operatorname{erfc}\left(\frac{\mathrm{E}\left[D_{i, u, A, B} \mid s_{i, u, B}=+1\right]}{\sqrt{2 \operatorname{Var}\left[D_{i, u, A, B} \mid s_{i, u, B}=+1\right]}}\right),
$$

which can be rewritten as

$$
\mathrm{BER}=\frac{1}{2} \operatorname{erfc}\left(\left[\frac{2 \operatorname{Var}\left[D_{i, u, A, B}\right]}{\mathrm{E}\left[D_{i, u, A, B}\right]^{2}}\right]^{-\frac{1}{2}}\right) .
$$

where $\operatorname{erfc}(x)$ is the complementary error function defined as the following

$$
\operatorname{erfc}(x) \equiv \frac{2}{\sqrt{\pi}} \int_{x}^{\infty} e^{-\mu^{2}} d \mu
$$


Using equations (34), (38), (40), and (42) in (45), the BER for the ANC MC-DCSK scheme can be determined by

$$
\mathrm{BER}=\frac{1}{2} \operatorname{erfc}\left(\left(\frac{2 E\left[W^{2}\right]+2 E\left[Z^{2}\right]+2 E\left[N^{2}\right]}{\mathrm{E}\left[D_{i, u, A, B}\right]^{2}}\right)^{\frac{-1}{2}}\right),
$$

or equivalently by

$$
\begin{aligned}
& \mathrm{BER}= \frac{1}{2} \operatorname{erfc}\left(\sum _ { v = 1 } ^ { V } \sum _ { v ^ { \prime } = 1 } ^ { V } \left(\frac{(M+1)\left(\lambda_{i, u, A, v^{\prime}}^{2}+1\right) N_{0}}{M \lambda_{i, u, A, v^{\prime}}^{2} \lambda_{i, u, B, v}^{2} \mathcal{E}_{b}^{(u)}}\right.\right. \\
&+ \frac{(M+1)\left(\lambda_{u, \Re_{A}, v^{\prime}}^{2}+1\right) N_{0} \sum_{\substack{l=1 \\
l \neq A \\
l \neq B}}^{L} \lambda_{i, u, A, l, v^{\prime}}^{2}}{M \lambda_{u, \Re_{A}, v^{\prime}}^{2} \lambda_{u, \Re_{B}, v}^{2} \mathcal{E}_{b}^{(u)}} \\
&\left.\left.+\frac{(M+1)^{2}\left(\lambda_{u, \Re_{A}, v^{\prime}}^{2}+1\right)\left(\lambda_{i, u, A, v}^{2}+1\right) N_{0}^{2}}{2 M^{2} \lambda_{u, \Re_{A}, v^{\prime}}^{2} \lambda_{u, \Re_{B}, v^{3} \lambda_{i, u, A, v^{\prime}}^{2} \lambda_{i, u, B, v}^{3} \mathcal{E}_{b}^{2(u)}}^{3}}\right)^{\frac{-1}{2}}\right) .
\end{aligned}
$$

The equation (48) gives the theoretical BER benchmark of the multi-user ANC MC-DCSK system over multipath fading channel. Based on this equation, it is important to point out that the channel varies from one bit to another, and that the average power gain of each path is different form the other ones as well as the other user's channels.

In terms of the instantaneous signal to noise ratios, equation (48) can notationally be represented by

$$
\mathrm{BER}=\frac{1}{2} \operatorname{erfc}\left(\sum_{v=1}^{V} \sum_{v^{\prime}=1}^{V}\left(\frac{1}{\gamma_{1}}+\frac{1}{\gamma_{2}}+\frac{1}{\gamma_{3} \gamma_{1}}\right)^{\frac{-1}{2}}\right),
$$

where $\gamma_{1}, \gamma_{2}$, and $\gamma_{3}$ represent the instantaneous signal to noise ratios given by

$$
\begin{gathered}
\gamma_{1}=\frac{M \lambda_{i, u, A, v^{\prime}}^{2} \lambda_{i, u, B, v}^{2} \mathcal{E}_{b}^{(u)}}{(M+1)\left(\lambda_{i, u, A, v^{\prime}}^{2}+1\right) N_{0}}, \\
\gamma_{2}=\frac{M \lambda_{u, \Re_{A}, v^{\prime}}^{2} \lambda_{u, \Re_{B}, v}^{2} \mathcal{E}_{b}^{(u)}}{(M+1) \sum_{\substack{l=1 \\
l \neq A \\
l \neq B}}^{L} \lambda_{i, u, A, v^{\prime}}^{2}\left(\lambda_{u, \Re_{A}, v^{\prime}}^{2}+1\right) N_{0}},
\end{gathered}
$$

and

$$
\gamma_{3}=\frac{2 M \lambda_{u, \Re_{A}, v^{\prime}}^{2} \lambda_{u, \Re_{B}, v}^{3} \mathcal{E}_{b}^{(u)}}{(M+1)\left(\lambda_{u, \Re_{A}, v^{\prime}}^{2}+1\right) N_{0}}=\sum_{\substack{l=1 \\ l \neq A \\ l \neq B}}^{L} \lambda_{i, u, A, v^{\prime}}^{2} \gamma_{2} .
$$

Equation (49) presents the general expression of BER as a function of instantaneous signal to noise ratios. Depending on the transmission scenario, for instance slow at fading channel or equal average power gain case, this equation can be more simplified.

Furthermore, the bit energy $\mathcal{E}_{b}^{(u)}$ that is of significant importance in equation (49), should not be considered as a constant parameter for all chaos-based spreading spectrum systems. Nevertheless, there have been many approaches to determine BER of chaos-based communication systems which use the Gaussian approximation, and consider the transmitted bit energy $\mathcal{E}_{b}^{(u)}$ as a constant parameter [50]. It is essential to note that this assumption is valid only for high spreading factors, but when it comes to low spreading factors the transmitted bit energy after being spread by the chaotic sequences, definitely varies from one bit to another [39], [51]. Therefore, the general BER expression for the multi-user ANC-based MC-DCSK scenario can be presented by

$$
\begin{gathered}
\operatorname{BER}_{t}=\iiint_{\gamma_{1}, \gamma_{2}, \gamma_{3}} \frac{1}{2} \operatorname{erfc}\left(\sum_{v=1}^{V} \sum_{v^{\prime}=1}^{V}\left(\frac{1}{\gamma_{1}}+\frac{1}{\gamma_{2}}+\frac{1}{\gamma_{3} \gamma_{1, i, u, v}}\right)^{\frac{-1}{2}}\right) \\
f\left(\gamma_{1}\right) f\left(\gamma_{2}\right) f\left(\gamma_{3}\right) d \gamma_{1} d \gamma_{2} d \gamma_{3},
\end{gathered}
$$

where $f(\lambda)$ is the probability density functions of the instantaneous signal-to-noise ratio. In some scenarios like flat fading channel, where the channel gain is constant within the used bandwidth, this equation becomes much simpler.

In $m$-distributed fading channels, the challenging task to obtain the BER expression theoretically, is to determine the integral in equation (53). Since the analytical derivation of the probability density functions in such scenarios, is generally nontrivial, it is common to compute the integral numerically.

\section{BER in AWGN Case}

For an AWGN channel, the coefficients $\lambda_{i, l, v}$ as well as the number of paths are constant and equal to one $(\lambda=1, V=$ 1). In this particular case, the analytical BER expression can be directly derived from (49)

$$
\mathrm{BER}_{t}=\frac{1}{2} \operatorname{erfc}\left(\frac{2(M+1) N_{0}}{M \mathcal{E}_{b}}+\frac{2(M+1) N_{0}(L-2)}{M \mathcal{E}_{b}}+\frac{2(M+1) N_{0}^{2}}{M \mathcal{E}_{b}^{2}}\right)^{\frac{-1}{2}} \text {. }
$$

\section{Simulation Results and Discussions}

In order to validate the performance of the ANC-based multi-user MC-DCSK scheme, the computed BER expressions are compared to the corresponding simulation results under an AWGN, and multipath fading channels (Rayleigh, and Rician scenarios). Figs. 7, 8, 9, and 10 show the obtained results for different numbers of subcarriers $M$, spreading factor $\beta$, average power gains, number of paths $V$, and number of users $L$.

Fig. 7 compares the computed BER using equation (54) with the Monte Carlo simulation results of the multi-user ANCbased MC-DCSK system under an AWGN channel. As can be seen the simulation results are perfectly validated by the computed BER for different numbers of subcarriers, and users. In order to show the effect of the number of subcarriers, the spreading factor $\beta$ and the number of users $L$ are set to 80 and 3, respectively, while the number of subcarriers $M$ varies from 2 to 32 . This means that for a given spreading factor, the reference energy needed to transmit one bit decrease as the number of subcarriers increases (i.e. see also equation (9)). This performance improvement is shown in Fig. 7, confirming that a higher number of subcarriers results in a lower energy needed to obtain a desired BER.

Fig. 8 demonstrates the performance enhancement of the ANC-based multi-user MC-DCSK system with and without 


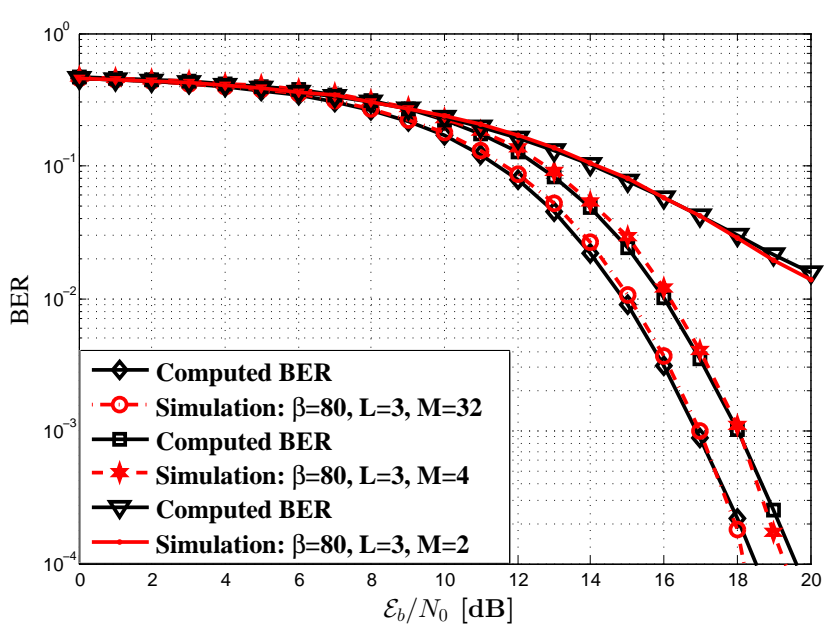

Fig. 7: Simulation and theoretical results of BER performance under an AWGN channel for different number of subcarriers $M$.

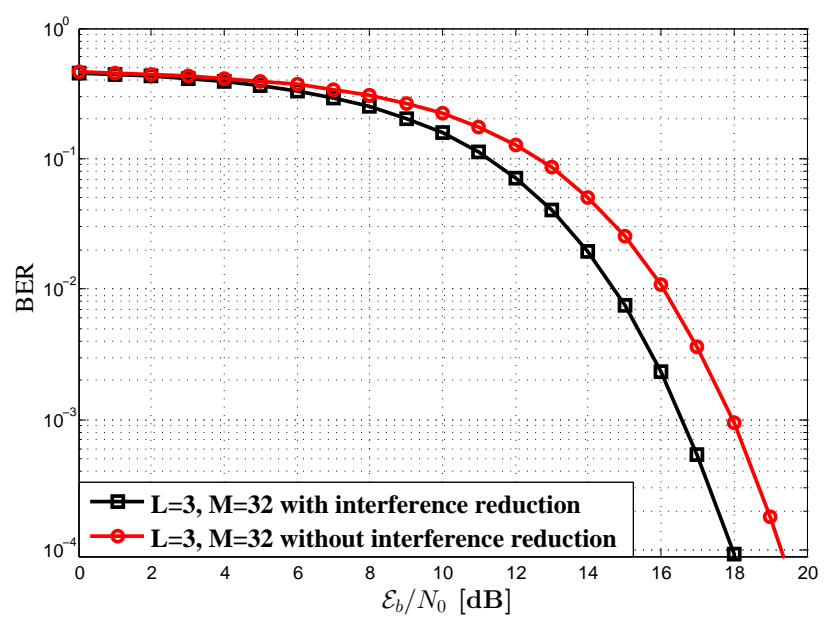

Fig. 8: The BER comparison of the ANC-based MC-DCSK scheme with and without self-interference cancellation under an AWGN channel.

self-interference cancellation, under an AWGN channel. The considerable enhancement of BER depicted in this figure is achieved by using self-interference cancellation technique for $L=3$ and $M=32$. Since each user eliminates just its own interference, without mitigating the interference caused by other signals coming from the other users, the performance improvement shown in this figure will be the same for any number of users. In other words, the gap between the two BER curves is independent of the number of the users. The figure shows the amount of the interference reduction in presence of additive white Gaussian noise which results in approximately $2 \mathrm{~dB}$ in enhancement BER.

Basically, when the number of users $L$ is more than 2, the performance of the system can be improved by applying successive or parallel interference cancellation (SIC and PIC) techniques which reduce the rest of the multi-user interferences caused by the $(L-2)$ users [52], [53]. However, these techniques require estimation of all channels and decoding of the $(L-2)$ data users to be able to reduce the impairment by multi-user interference. The performance enhancement in this case will be at the expense of hardware and computation complexity. Since the application of such techniques is only concerned with the cost, most of users are generally satisfied to pay for a higher performance of the system.

It is crucial to point out that in this system, the application of inherently uncorrelated chaotic signals leads to lower multiuser interferences compared to that of the conventional multiuser spread spectrum systems. Therefore, it is safe to claim that the proposed scheme is a simple but intelligent approach to eliminating the self-interference of a given user that leads to good results with low complexity and low computation cost.

Figs. 9 and 10 depict the comparison between the numerically computed bit error rate given in equation (53) and the corresponding simulation results. These two figures illustrate the effect of the multi-path Rayleigh and Rician channels on the performance of the ANC-based MC-DCSK system. The

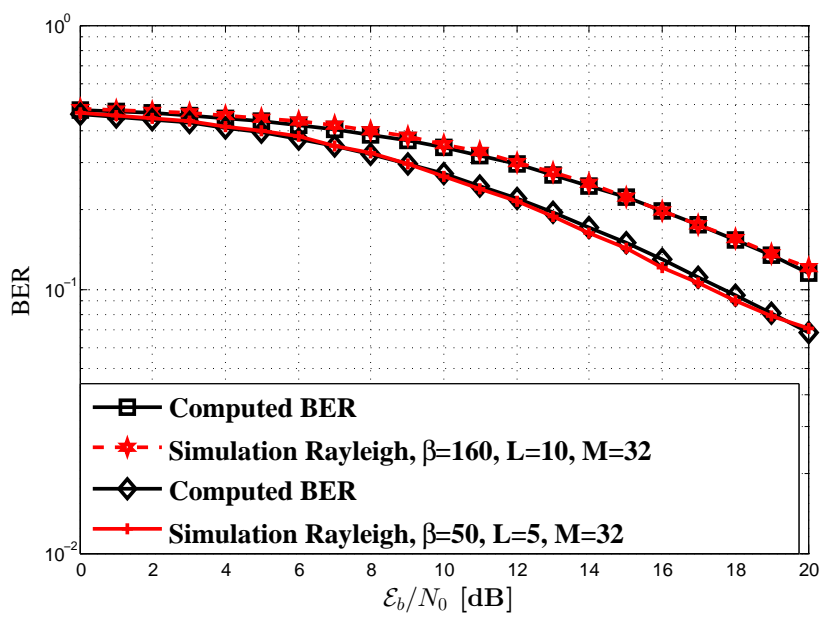

Fig. 9: Simulation and theoretical expression results of BER performance under multipath Rayleigh fading channel for the number of users $L=5, L=10$ and the spreading factors $\beta=50, \beta=160$, with the number of subcarriers $M=32$ and the number of paths $V=3$.

obtained BER performance under multipath Rayleigh fading channel in Fig. 9 corresponds to the number of users $L=5$ and $L=10$, the spreading factors of $\beta=50$ and $\beta=160$, with the number of subcarriers $M=32$ and the number of the paths $V=3$. The average power gain of each path is identical for all users in the network. In this figure, the average power gains are $\mathrm{E}\left[\lambda_{1}^{2}\right]=0.9$ for the line-of-sight, $\mathrm{E}\left[\lambda_{2}^{2}\right]=0.8$ for the second path, and $\mathrm{E}\left[\lambda_{3}^{2}\right]=0.65$ for the third path. The delay spread $\tau_{l, v}$ for each path is denoted by a random variable with uniform distribution, taking values within the interval $\left[0,5 T_{c}\right]$. The simulation results validate the accuracy of the proposed methodology.

Fig. 10 summarizes the the simulation and theoretical results of BER performance under the multipath Rician fading channel corresponding to the number of users $L=5$ and $L=6$, the spreading factors of $\beta=50$ and $\beta=100$, with the number of subcarriers $M=32$ and the number of the paths $V=2$. The average power gain for the line-of-sight path is 
$\mathrm{E}\left[\lambda_{1}^{2}\right]=0.95$, while for the second path is $\mathrm{E}\left[\lambda_{2}^{2}\right]=0.75$. According to this figure, the simulation results closely follow the computed BER under the Rician channel for all number of subcarriers, average power gains, spreading factors, and for all number of users.

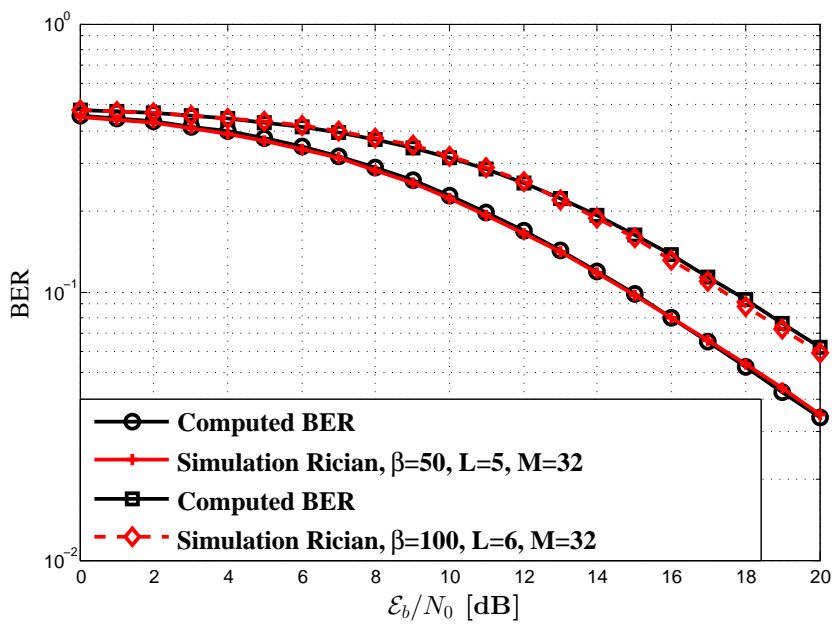

Fig. 10: Simulation and theoretical expression results of BER performance under multipath Rician fading channel for the number of users $L=5, L=6$ and the spreading factors of $\beta=50, \beta=100$, with the number of subcarriers $M=32$ and the number of paths $V=2$.

Finally, Fig. 11 shows the simulation results of the bit error rate performance for the general ANC-based DCSK and the proposed ANC-based MC-DCSK. The results are obtained under the multipath Rician fading channel for the spreading factor of $\beta=50$ and the number of paths $V=2$. The average power gain for the line-of-sight path is $\mathrm{E}\left[\lambda_{1}^{2}\right]=0.95$, whereas for the second path is $\mathrm{E}\left[\lambda_{2}^{2}\right]=0.75$. Furthermore, in the MCDCSK system, the number of subcarriers is $M=32$ with $L=2$ and $L=5$ users. Based on the simulation results depicted in this figure, it can be concluded that the number of users in the general ANC-based DCSK system is limited to $L=2$ due to the lack of capacity for supporting the communications between more than two users. In contrast, the MC-DCSK scheme outperforms the conventional DCSK scheme even for a higher number of users such as $L=5$. The poor performance of the conventional DCSK system is mainly attributed to the strong interference originated from the cross product of the user data signals and the reference signals present in its decision variable given in equation (6). Therefore, the conventional form of DCSK system can not be considered as a promising candidate for an ANC application without any interference mitigation technique.

\section{CONCLUSION}

An analog network coding has been proposed for the first time for a multi-user MC-DCSK transmitter. One of the major objectives to choose MC-DCSK system in this paper is that its novel design allows fora more significant energy saving and higher spectral efficiency compared to that of the differential DCSK system. In the proposed network, the $L$

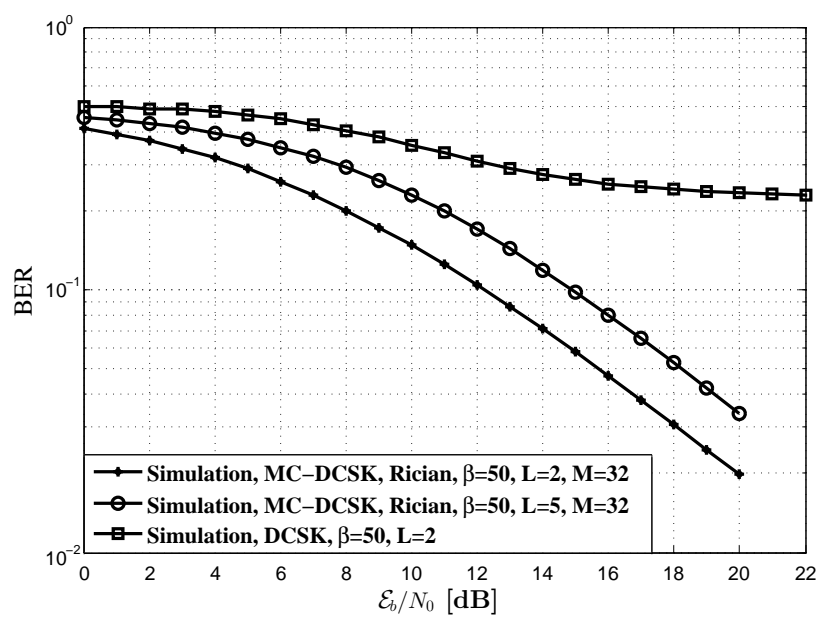

Fig. 11: Simulation results of BER performance for MCDCSK and DCSK schemes under multipath Rician fading channel for the number of users $L=5$, and $L=2$ and the spreading factor of $\beta=50$ and the number of paths $V=2$.

source nodes communicate with each other via a single relay node. The ANC scheme is developed in a way that the user nodes transmit their signals to the relay in the first time slot synchronously or asynchronously. During the second time slot, the relay forwards the resulting superimposed signal to the users. In order to use the available bandwidth efficiently, the MC-DCSK is applied to a multi-user system which is suitable for ANC scheme. Each user is assigned a private frequency for transmitting the reference signal while they share the rest of the available bandwidth for transmitting their spread binary data to the other users in the network. At the receiver side which benefits from non-coherent detection, each user reduces the overall interference by subtracting its own data signal from the received signal and then starts the decoding process. Unlike DCSK scheme, it was also shown that the proposed system can perform with more than two users. Finally, the performance of the ANC-based MC-DCSK was analyzed, and the general bit error rate expression under multipath fading channels was obtained, and then extended to an additive white Gaussian noise scenario. The Simulation results were validated by the results obtained from the corresponding theoretical expression.

\section{REFERENCES}

[1] K. David, D. Dixit, and N. Jefferies, "2020 Vision,” IEEE Vehicular Technology Mag., vol. 5, no. 3, pp. 22 -29, 2010.

[2] S. Katti, R. S. Gollakota, and D. Katabi, "Embracing Wireless Interference: Analog Network Coding," in Proc. ACM SIGCOMM, 2007, pp. $397-408$.

[3] M. Riemensberger, Y. Sagduyu, M. Honig, and W. Utschick, "Comparison of Analog and Digital Relay Methods With Network Coding for Wireless Multicast," in IEEE International Conference on Communications, ICC '09, 2009, pp. 1-5.

[4] I. Maric, A. Goldsmith, and M. Medard, "Analog Network Coding in The High-SNR Regime," in IEEE Wireless Network Coding Conference (WiNC), 2010, pp. 1-6.

[5] S. C. Liew, S. Zhang, and L. Lu, "Physical-Layer Network Coding: Tutorial, Survey, and Beyond," CoRR, vol. abs/1105.4261, 2011.

[6] S. Mao, J. Kim, and J. Lee, "Multi-User Analog Network Coding With Spread Spectrum," in IEEE 75th Vehicular Technology Conference (VTC Spring), 2012, pp. 1-5. 
[7] H. Kulhandjian, T. Melodia, and D. Koutsonikolas, "CDMA-Based Analog Network Coding Through Interference Cancellation for Underwater Acoustic Sensor Networks," in The Seventh ACM International Conference on Underwater Networks and Systems, ser. WUWNet '12. New York, NY, USA: ACM, 2012, pp. $1-8$.

[8] M. Chen and A. Yener, "Multiuser Two-Way Relaying: Detection and Interference Management Strategies," IEEE Trans. on Wireless Communications, vol. 8, no. 8, pp. 4296 - 4305, 2009.

[9] F. Escribano, L. Lopez, and M. Sanjuan, "Improving The Performance of Chaos-Based Modulations Via Serial Concatenation," IEEE Trans. on Circuits and Systems I: Regular Papers, vol. 57, no. 2, pp. 448-459, 2010.

[10] F. Escribano and A. Tarable, "Interleaver Design for Parallel Concatenated Chaos-Based Coded Modulations," IEEE Communications Letters, vol. 17, no. 5, pp. $834-837,2013$.

[11] F. C. M. Lau and C. K. Tse, Chaos-Based Digital Communication Systems. Springer-Verlag, 2003.

[12] A. P. Kurian, S. Puthusserypady, and S. M. Htut, "Performance Enhacment of DS-CDMA System Using Chaotic Complex Spreading Sequence," IEEE Trans. on Wireless Communications, vol. 4, no. 3, pp. $984-989,2005$.

[13] R. Vali, S. Berber, and S. K. Nguang, "Accurate Derivation of ChaosBased Acquisition Performance in a Fading Channel," IEEE Trans. Wireless Communications, vol. 11, no. 2, pp. 722-731, 2012.

[14] R. Vali, S. Berber, and S.-K. Nguang, "Analysis of Chaos-Based Code Tracking Using Chaotic Correlation Statistics," IEEE Trans. on Circuits and Systems I: Regular Papers, vol. 59, no. 4, pp. 796 - 805, 2012.

[15] S. Berber and S. Feng, "Chaos-Based Physical Layer Design for WSN Applications," in Recent Advances in Telecommunications and Circuit Design (CIRCOM 2013), vol. 2, 2013, pp. 157 - 162.

[16] Y. Xia, C. K. Tse, and F. C. M. Lau, "Performance of Differential ChaosShift-Keying Digital Communication Systems over a Multipath Fading Channel with Delay Spread," IEEE Trans. on Circuits and Systems II, vol. 51, pp. $680-684,2004$.

[17] J. Yu and Y.-D. Yao, "Detection Performance of Chaotic Spreading LPI Waveforms," IEEE Trans. on Wireless Communications, vol. 4, no. 2, pp. 390 - 396, 2005.

[18] V. Lynnyk and S. Celikovsky, "On The Anti-Synchronization Detection for The Generalized Lorenz System and Its Application to Secure Encryption," International Journal published by Institute of Information Theory and Automation, vol. 46, pp. 1-18, 2010.

[19] S. Vitali, R. Rovatti, and G. Setti, "Improving PA Efficiency by ChaosBased Spreading in Multicarrier DS-CDMA Systems," in IEEE International Symposium on Circuits and Systems, (ISCAS), 2006, pp. 1194 $-1198$.

[20] G. Mazzini, G. Setti, and R. Rovatti, "Chaotic Complex Spreading Sequences for Asynchronous DS-CDMA Part I: System Modeling and Results," IEEE Trans. on Circuits and Systems I: Fundamental Theory and Applications, vol. 44, pp. 937 - 1947, 1997.

[21] F. Lau and C. Tse, "Optimum Correlator-Type Receiver Design for CSK Communication Systems," Int. J. Bifurcation chaos, vol. 5, pp. 1029 $1038,2002$.

[22] G. Kolumbau, "Theoretical Noise Performance of Correlator-Based Chaotic Communications Schemes," IEEE Trans. on Circuits and Systems I: Fundamental Theory and Applications, vol. 47, no. 12, pp. 1692 1701, 2000.

[23] S. Arai, Y. Nishio, and T. Yamazato, "Error-Correcting Scheme Based on Chaotic Dynamics and Its Performance for Noncoherent Chaos Communications," IEICE Nonlinear Theory and Its Applications, vol. 1, no. 1 , pp. $196-206,2010$.

[24] G. Kaddoum, J. Olivain, G. Beaufort Samson, P. Giard, and F. Gagnon, "Implementation of a Differential Chaos Shift Keying Communication System in GNU Radio," in International Symposium on Wireless Communication Systems (ISWCS), 2012, pp. 934 - 938.

[25] G. Kolumbán, G. Kis, Z. Jákó, and M. P. Kennedy, "FM-DCSK A Robust Modulation Scheme for Chaotic Communications," IEICE Trans. on Fundamentals of Electronics, Communications and Computer, vol. 89, pp. 1798 - 1802, 1998.

[26] R.-R. Chen, R. Koetter, U. Madhow, and D. Agrawal, "Joint Noncoherent Demodulation and Decoding for The Block Fading Channel: A Practical Framework for Spproaching Shannon Capacity," IEEE Trans. on Communications, pp. 1676 - 1689, 2003.

[27] B. Le Saux, M. Helard, and P.-J. Bouvet, "Comparison of Coherent and Non-Coherent Space Time Schemes for Frequency Selective FastVarying Channels," in International Symposium on Wireless Communication Systems (ISWCS), 2005, pp. $32-36$.
[28] G. Kaddoum, F. Gagnon, P. Charge, and D. Roviras, "A Generalized BER Prediction Method for Differential Chaos Shift Keying System Through Different Communication Channels," Wireless Personal Communications, vol. 64, pp. 425 - 437, 2012.

[29] Y. Fang, L. W. J. Xu, and G. Chen, "Performance of MIMO Relay DCSK-CD Systems over Nakagami Fading Channels," IEEE Trans. on Circuits and systems I, vol. 60, pp. 1-11, 2013.

[30] Y. Fang, L. Wang, and G. Chen, "Performance of a Multiple-Access DCSK-CC System over Nakagami-m Fading Channels," in IEEE International Symposium on Circuits and Systems (ISCAS), 2013, pp. $277-$ 280.

[31] W. Xu, L. Wang, and G. Chen, "Performance of DCSK Cooperative Communication Systems over Multipath Fading Channels," IEEE Trans. on Circuits and Systems I: Regular Papers, vol. 58, no. 1, pp. 196 -204, 2011.

[32] G. Kaddoum, F. Gagnon, and F.-D. Richardson, "Design of a Secure Multi-Carrier DCSK System," in The ninth international symposium on wireless communication systems (ISWCS), 2012, pp. 964 - 968.

[33] G. Cimatti, R. Rovatti, and G. Setti, "Chaos-Based Spreading in DSUWB Sensor Networks Increases Available Bit Rate," IEEE Trans. on Circuits and Systems I: Regular Papers, vol. 54, no. 6, pp. 1327 - 1339, 2007.

[34] C.-C. Chong and S. K. Yong, "UWB Direct Chaotic Communication Technology for Low-Rate WPAN Applications," IEEE Trans. on Vehicular Technology, vol. 57, no. 3, pp. 1527 - 1536, 2008.

[35] X. Min, W. Xu, L. Wang, and G. Chen, "Promising Performance of a Frequency-Modulated Differential Chaos Shift Keying Ultra-Wideband System Under Indoor Environments," IET Communications, vol. 4, no. 2, pp. $125-134,222010$.

[36] H. Yang and G.-P. Jiang, "High-Efficiency Differential-Chaos-ShiftKeying Scheme for Chaos-Based Noncoherent Communication," IEEE Trans. Circuits and Systems II, vol. 59, no. 5, pp. 312 - 316, 2012.

[37] W. K. Xu, L. Wang, and G. Kolumban, " A Novel Differential Chaos Shift Keying Modulation," International Journal of Bifurcation and Chaos, vol. 21, no. 03, pp. 799 - 814, 2011.

[38] G. Kaddoum and F. Gagnon, "Design of a High-Data-Rate Differential Chaos-Shift Keying System," IEEE Trans. on Circuits and Systems II, vol. 59, no. 99, pp. 1-5, 2012.

[39] G. Kaddoum, F. Richardson, and F. Gagnon, "Design And Analysis of a Multi-Carrier Differential Chaos Shift Keying Communication System," IEEE Trans. on Communications, vol. 61, no. 8, pp. 3281 - 3291, 2013.

[40] G. Kaddoum, F.-D. Richardson, S. Adouni, F. Gagnon, and C. Thibeault, "Multi-user multi-carrier differential chaos shift keying communication system," in International Wireless Communications and Mobile Computing Conference (IWCMC), 2013, pp. 1798 - 1802.

[41] T. S. Rappaport, Wireless Communications: Principles and Practice. Englewook Cliffs, NJ: Prentice-Hall, 1996.

[42] P. Chen, L. Wang, and F. Lau, "One Analog STBC-DCSK Transmission Scheme Not Requiring Channel State Information," IEEE Trans. on Circuits and Systems I: Regular Papers, vol. 60, no. 4, pp. 1027 - 1037, 2013.

[43] K. Yu and I. Oppermann, "Performance of Decorrelating Receivers in Multipath Rician Fading Channels," IEEE Trans. on Wireless Communications, vol. 5, no. 8, pp. 2009 - 2016, 2006.

[44] J. G. Proakis, Digital communications. mcgraw, 2001.

[45] J. Evans and D. Tse, "Large System Performance of Linear Multiuser Receivers in Multipath Fading Channels," IEEE Trans. on Information Theory, vol. 46, no. 6, pp. 2059 - 2078, Sep 2000.

[46] R. L. Peterson, R. E. Zeimer, and D. E. Borth, Introduction to Spread Spectrum Communications. Prentice Hall International, 1995.

[47] S. Kondo and B. Milstein, "Performance of Multicarrier DS-CDMA Systems," IEEE Trans. Communications, vol. 44, no. 2, pp. 238 - 246, 1996.

[48] G. Kolumbán and G. Kis, "Multipath Performance of FM-DCSK Chaos Communications System," in International symposium of circuits and systems, ISCAS, 2000, pp. $433-436$.

[49] K. Umeno and K. Kitayama, "Spreading Sequences Using Periodic Orbits of Chaos for CDMA," Electronics Letters, vol. 35, no. 7, pp. 545 - 546, 1999.

[50] M. Sushchik, L. S. Tsimring, and A. R. Volkovskii, "Performance Analysis of Correlation-Based Communication Schemes Utilizing Chaos," IEEE Trans. on Circuits and Systems I, vol. 47, pp. 1684 - 1691, 2000.

[51] G. Kaddoum, P. Chargé, and D. Roviras, "A Generalized Methodology for Bit-Error-Rate Prediction in Correlation-Based Communication Schemes Using Chaos," IEEE Commun. Letters, vol. 13, no. 8, pp. 567 $-569,2009$. 
[52] P. Patel and J. Holtzman, "Analysis of a Simple Successive Interference Cancellation Scheme in a DS/CDMA System," IEEE Journal on Selected Areas in Communications, vol. 12, no. 5, pp. 796 - 807, 1994.

[53] M. Ghotbi and M. Soleymani, "Multiuser Detection of DS-CDMA Signals Using Partial Parallel Interference Cancellation in Satellite Communications," IEEE Journal on Selected Areas in Communications, vol. 22, no. 3, pp. $584-593,2004$.

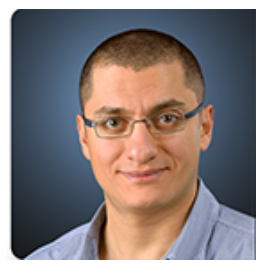

Georges Kaddoum is an assistant Professor of Electrical Engineering at École De Technologie Supérieure, University of Québec, Montreal, Canada. Dr. Kaddoum received his B.Sc. degree in Electrical Engineering from ENSTA-Bretagne (École nationale supérieure de techniques avancées) and M.S. degree in Telecommunications and Signal Processing (circuits, systems and signal processing) from Université de Bretagne Occidentale and Telecom Bretagne (Brest, France) in September 2005. In December 2008, he completed his Ph.D. in signal processing and telecommunications with honor from the National Institute of Applied Sciences (INSA), University of Toulouse, France. From September 2009 to October 2012, he was served as postdoctoral fellow at Laboratoire de communication et d'intégration de la microélectronique (LACIME), ÉTS. He was recognized as an institutional researcher in 2012 and then promoted as an assistant professor in November 2013. His recent research activities cover wireless communication systems, chaotic modulations, secure transmissions, and space communications and navigation. He has published over 50 journal and conference papers and held two pending patents. He is currently an editorial board of the CIP Journal Wireless Communications and Networking. Dr. Kaddoum is also a scientific consultant in the field of space telecommunications for Intelcan Techno-systems and MDA corporation companies.

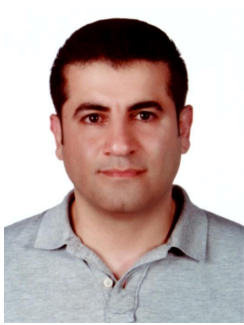

Farhad Shokraneh received the M.Sc. degree in communication systems from Lund University, Lund, Sweden, in 2012. From January to July 2013, He worked as Research Assistant at the Department of Electric and Information Technology (EIT), Lund University. He is presently a $\mathrm{PhD}$ student in wireless communications at Laboratoire de communication et d'intégration de la microélectronique (LACIME), École De Technologie Supérieure, University of Quebec, Montreal, Canada. His current research interests are wireless networks, chaos-based communications, and random number generators. 